\title{
BMJ Open Incidence, clinical characteristics and long-term prognosis of postoperative symptomatic venous thromboembolism: a retrospective cohort study
}

\author{
Chikashi Takeda (D) , ${ }^{1}$ Yugo Yamashita, ${ }^{2}$ Masato Takeuchi, ${ }^{3}$ Hiroshi Yonekura (D) , \\ Li Dong, ${ }^{1}$ Miho Hamada, ${ }^{1}$ Akiko Hirotsu, ${ }^{1}$ Koh Ono, ${ }^{2}$ Koji Kawakami (D) , ${ }^{3}$ \\ Kazuhiko Fukuda, ${ }^{1}$ Takeshi Morimoto (D) , ${ }^{2}$ Takeshi Kimura (D) , ${ }^{2}$ \\ Toshiyuki Mizota (D) ${ }^{1}$
}

To cite: Takeda C, Yamashita Y, Takeuchi M, et al. Incidence, clinical characteristics and long-term prognosis of postoperative symptomatic venous thromboembolism: a retrospective cohort study. BMJ Open 2022;12:e055090. doi:10.1136/ bmjopen-2021-055090

- Prepublication history and additional supplemental material for this paper are available online. To view these files, please visit the journal online (http://dx.doi.org/10.1136/ bmjopen-2021-055090).

Received 03 July 2021 Accepted 26 January 2022
Check for updates

(C) Author(s) (or their employer(s)) 2022. Re-use permitted under CC BY-NC. No commercial re-use. See rights and permissions. Published by BMJ.

For numbered affiliations see end of article.

Correspondence to Dr Toshiyuki Mizota; mizota@kuhp.kyoto-u.ac.jp

\section{ABSTRACT}

Objectives The purpose of this study was to evaluate the incidence, clinical characteristics and prognosis of postoperative symptomatic venous thromboembolism (VTE) in Japan.

Design Retrospective observational study. Two data sets, Contemporary ManageMent AND outcomes in patients with Venous ThromboEmbolism (COMMAND VTE) Registry and Japanese Society of Anesthesiologists (JSA) annual report, were used for current analyses.

Setting Eighteen of 29 centres participated in the COMMAND VTE Registry.

Participants Acute symptomatic patients with VTE who had undergone surgery 2 months prior to the diagnosis at 18 centres from January 2010 to December 2013 were identified in the COMMAND VTE Registry. From each centre's JSA annual report, the overall population that had received anaesthetic management during this period was retrieved. Interventions None.

Primary and secondary outcome measures The primary outcome was the incidences and clinical characteristics of postoperative symptomatic VTE. The secondary outcomes were recurrent VTE, major bleeding and all-cause death.

Results We identified 137 patients with postoperative symptomatic VTE, including 57 patients with pulmonary embolism. The incidences of postoperative symptomatic VTE and pulmonary embolism were $0.067 \%$ and $0.028 \%$, respectively, based on data from 203943 patients who underwent surgery, managed by anaesthesiologists, during the study period. The incidences of postoperative symptomatic VTE varied widely, depending on surgical and anaesthetic characteristics. Postoperative symptomatic VTE occurred at a median of 8 days after surgery, with 58 patients (42\%) diagnosed within 7 days. The cumulative incidence, 30 days after VTE, of recurrent VTE, major bleeding, and all-cause death was $3.0 \%, 5.2 \%$, and $3.7 \%$, respectively.

Conclusion This study, combining the large real-world VTE and anaesthesiology databases in Japan revealed the incidence, clinical features and prognosis of postoperative symptomatic VTE, providing useful insights for all healthcare providers involved in various surgeries.

\section{Strengths and limitations of this study}

- Venous thromboembolism (VTE) is considered relatively rare in Asian people, and the small number of cases makes epidemiological studies difficult to perform.

- This study combines data from the large real-world VTE database and anaesthetic database in Japan to provide information about the incidence, clinical features and prognosis of postoperative symptomatic VTE.

- Another important feature of the current study was the comparison of the incidence of postoperative symptomatic VTE across surgical sites.

- This was a retrospective cohort study with inherent limitations based on its observational nature. Furthermore, as a certain number of patients from ineligible centres were excluded, the incidence of postoperative symptomatic VTE may have been influenced.

Trial registration Not applicable.

\section{INTRODUCTION}

Venous thromboembolism (VTE), including pulmonary embolism (PE) and deep vein thrombosis (DVT), is a serious postoperative complication, which can result in an in-hospital death. ${ }^{12}$ In perioperative management, it is crucial to prevent postoperative symptomatic VTE and to respond promptly, once it is recognised. Therefore, clinicians should be familiar with the clinical features of postoperative symptomatic VTE to optimise their management strategies.

Over the past 20 years, several guidelines have been recommended for the prophylaxis of postoperative VTE. ${ }^{3-5}$ Despite the use of preventive measures, the incidence of postoperative VTE remains high and varies 
from $0.58 \%$ to $2.2 \%$, according to reports from Western countries ${ }^{6-8}$ Furthermore, the incidence rate of symptomatic VTE in patients after spinal surgery for metastases in the spine has been reported to be substantially higher $(11 \%) .{ }^{9}$ However, data on postoperative VTE from a cohort/registry-based study in Asian countries are scarce. A previous study reported a relatively low incidence $(0.031 \%)$ of postoperative VTE throughout Japan. ${ }^{10}$ However, it was a surveillance study of postoperative PE, conducted by mailing questionnaires to anaesthesiologists; therefore, the possibility of under-reporting of events cannot be denied. Although the incidence of VTE in Asia has been considered to be lower than Western countries, ${ }^{11}$ recent studies have suggested an underestimation of VTE in Asia. ${ }^{12-14}$ No large-scale study has systematically evaluated the incidence of postoperative symptomatic VTE in Japan.

Therefore, with a collaborative effort between cardiologists and anaesthesiologists, we investigated the incidence, clinical characteristics and prognosis of postoperative symptomatic VTE, using a large, observational, real-world VTE database and an anaesthetic database of annual reports submitted to the Japanese Society of Anesthesiologists (JSA).

\section{METHODS}

\section{Study design, setting and population}

In this study, two data sets were used for analyses. The first was Contemporary ManageMent AND outcomes in patients with Venous ThromboEmbolism (COMMAND VTE) registry, a retrospective multicentre cohort study, which provided the data on patients with postoperative symptomatic VTE. The second was the JSA annual report, which provided cross-sectional data of all patients, who underwent surgical operations, managed by anaesthesiologists.

The design of the COMMAND VTE Registry has been reported in detail elsewhere. ${ }^{15}$ Briefly, this physicianinitiated registry was a large cohort of consecutive patients with acute symptomatic VTE, who were objectively confirmed by the cardiologists at 29 centres in Japan, between January 2010 and August 2014. In this registry, the hospital databases were searched for clinical diagnoses and imaging examinations of patients with suspected VTE, and consecutive patients who met the definition of acute symptomatic VTE were enrolled. Baseline data were obtained from the hospital charts or hospital databases. Follow-up data on vital status, recurrent VTE, bleeding and status of anticoagulation therapy, according to the prespecified definitions, were collected from the hospital charts, hospital databases or by contacting patients, relatives and/or referring to physicians through phone and/ or mail.

As for the JSA annual reports, the training hospitals certified by the JSA are required to submit the annual reports to the JSA at the end of the year, which includes the total number of surgeries managed by anaesthesiologists, patient characteristics in detail and surgical and anaesthetic information.

In this study, the JSA annual reports from January 2010 to December 2013 were collected from 18 centres that participated in the COMMAND VTE Registry. Furthermore, additional data of patients with postoperative symptomatic VTE, namely operative date, operative procedure, surgical sites, surgical position and types of anaesthesia on anaesthetic charts at each centre, were obtained.

In this study, patients from the 18 centres registered in the COMMAND VTE Registry, for which the JSA annual report was collected between January 2010 and December 2013, were enrolled (figure 1). We could not enrol patients from the remaining 11 centres of the COMMAND VTE Registry as their JSA annual reports and/or additional data on patients with postoperative symptomatic VTE were unavailable. We also could not register the patients between January 2014 and August 2014, since the JSA annual report was from January to December of each year. Furthermore, within the COMMAND VTE Registry, cases of symptomatic postoperative VTE within 2 months ${ }^{16}$ were identified. The overall population that had received anaesthetic management, during the study period, was retrieved from each centre's JSA annual report. Besides, additional data of patients with postoperative symptomatic VTE, namely surgery date, surgical procedure, surgical site, surgical position and type of anaesthesia on anaesthetic charts at each centre, were obtained.

\section{Patient and public involvement statement}

Patients or the public were not involved in the design, conduct, reporting or dissemination plans of our research.

\section{Definition of postoperative symptomatic VTE}

In this study, postoperative symptomatic VTE was defined as a thromboembolic event that occurred within 2 months of the postoperative period. ${ }^{16}$ The symptoms of VTE were defined as sudden-onset dyspnoea, pleuritic and substernal chest pain, cough, fever, haemoptysis and syncope for PE; and erythema, warmth, pain, swelling, tenderness and pain on dorsiflexion of the foot for DVT. Additionally, a sudden onset of abnormality in the vital signs, such as a decrease in arterial oxygen saturation and hypotension, was considered as symptoms of PE.

\section{Collection of baseline patient characteristics and clinical follow-up data}

In the COMMAND VTE Registry, data for the patients' characteristics were collected from the hospital charts or hospital databases, according to the prespecified definitions, using an electronic case report form in a web-based database system. Physicians at each of the institutions were responsible for data entry, and data were automatically examined for missing or contradictory input and out-of-range values. Additional edits were performed at the general office of the registry.

Patients with postoperative symptomatic VTE, identified through the COMMAND VTE Registry, were 


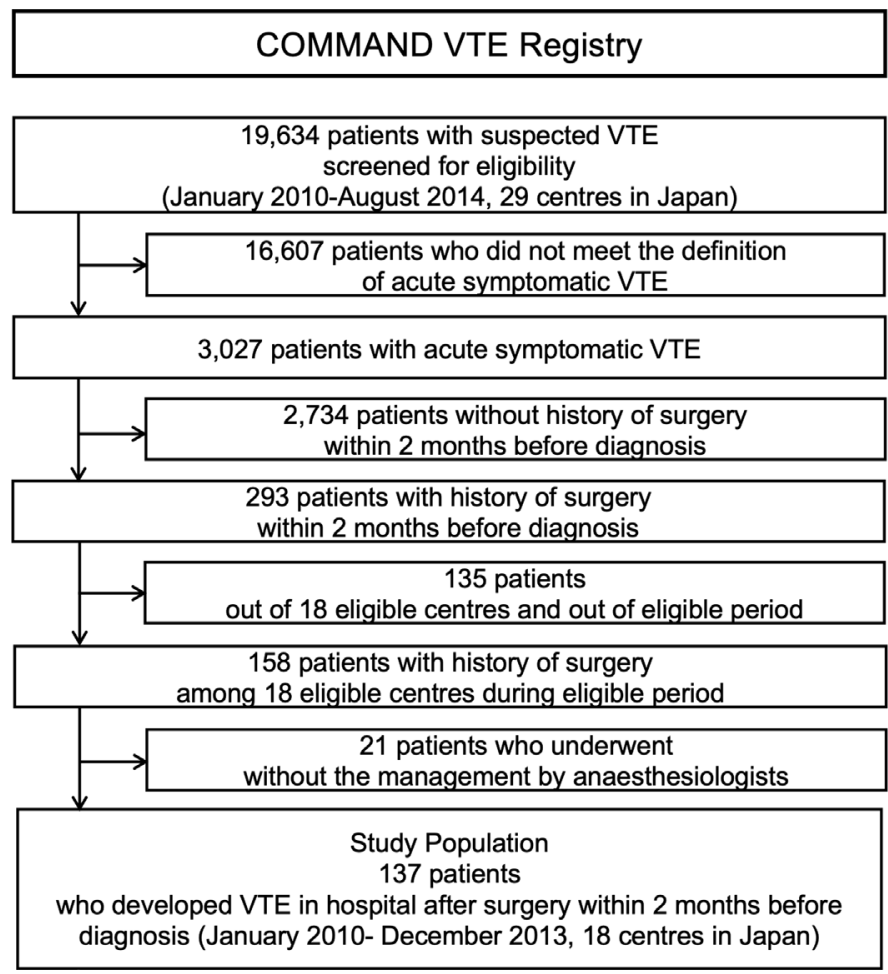

Annual reports to JSA from each centre

Figure 1 Study flow diagram. COMMAND VTE, Contemporary management and outcomes in patients with venous thromboembolism; DVT, deep vein thrombosis; JSA, Japanese Society of Anesthesiologists; PE, pulmonary embolism; VTE, venous thromboembolism.

further investigated at each centre using the anaesthetic charts created through the collaboration of cardiologists and anaesthesiologists at each participating centre. Anaesthesia-associated data, such as surgical site, surgical position and type of anaesthesia, were extracted and incorporated into the data from the COMMAND VTE Registry.

The outcome measures assessed in this study were recurrent VTE, major bleeding and all-cause death during the follow-up period, with a median of 1507 days, in the surviving patients. Recurrent VTE was defined as symptomatic PE and/or DVT accompanied by confirmation of a new thrombus or exacerbation of the thrombus by objective imaging examinations or autopsy. Major bleeding was defined according to the International Society of Thrombosis and Hemostasis as a reduction in the haemoglobin level by at least $2 \mathrm{~g} / \mathrm{dL}$, transfusion of at least two units of blood, or symptomatic bleeding in a critical area or an organ. ${ }^{17}$

\section{Statistical analysis}

The incidence of postoperative symptomatic VTE was calculated using a combination of data from the COMMAND Registry and the JSA annual reports from the 18 centres. The numerator of the incidence was the number of cases of postoperative symptomatic VTE extracted from the COMMAND Registry; the denominator was the number of surgeries in the JSA annual report. The incidence of postoperative symptomatic VTE according to age, sex, surgical site, surgical position and type of anaesthesia was calculated. The baseline and follow-up data were separately recorded for PE with or without DVT and DVT-only groups in patients with postoperative symptomatic VTE. No imputation was performed for missing data. Categorical variables were calculated as numbers and percentages, and continuous variables were calculated as the means and SD or the medians and IQR based on their distributions. Additionally, the timing of the postoperative symptomatic VTE occurrence after the surgery was described. The Kaplan-Meier method was used to estimate the cumulative incidences of recurrent VTE, major bleeding and all-cause death. The log-rank test was used to assess the differences in the cumulative incidences of the events between the PE-only and DVTonly groups. In addition, we conducted an exploratory analysis to compare patients with and without active cancer. Two-sided $p$ values of less than 0.05 were considered significant. All statistical analyses were performed using SAS V.9.4 for Windows (SAS Institute; Cary, North Carolina) or JMP V.14.0.0 (SAS Institute; Cary, North Carolina).

\section{RESULTS}

Figure 1 represents the flow diagram of the study. We enrolled 3027 consecutive patients with acute symptomatic VTE, after screening 19634 consecutive patients with suspected VTE for eligibility, using the chart review by the physicians at each institution. After excluding 2734 
Table 1 Incidence of postoperative symptomatic VTE

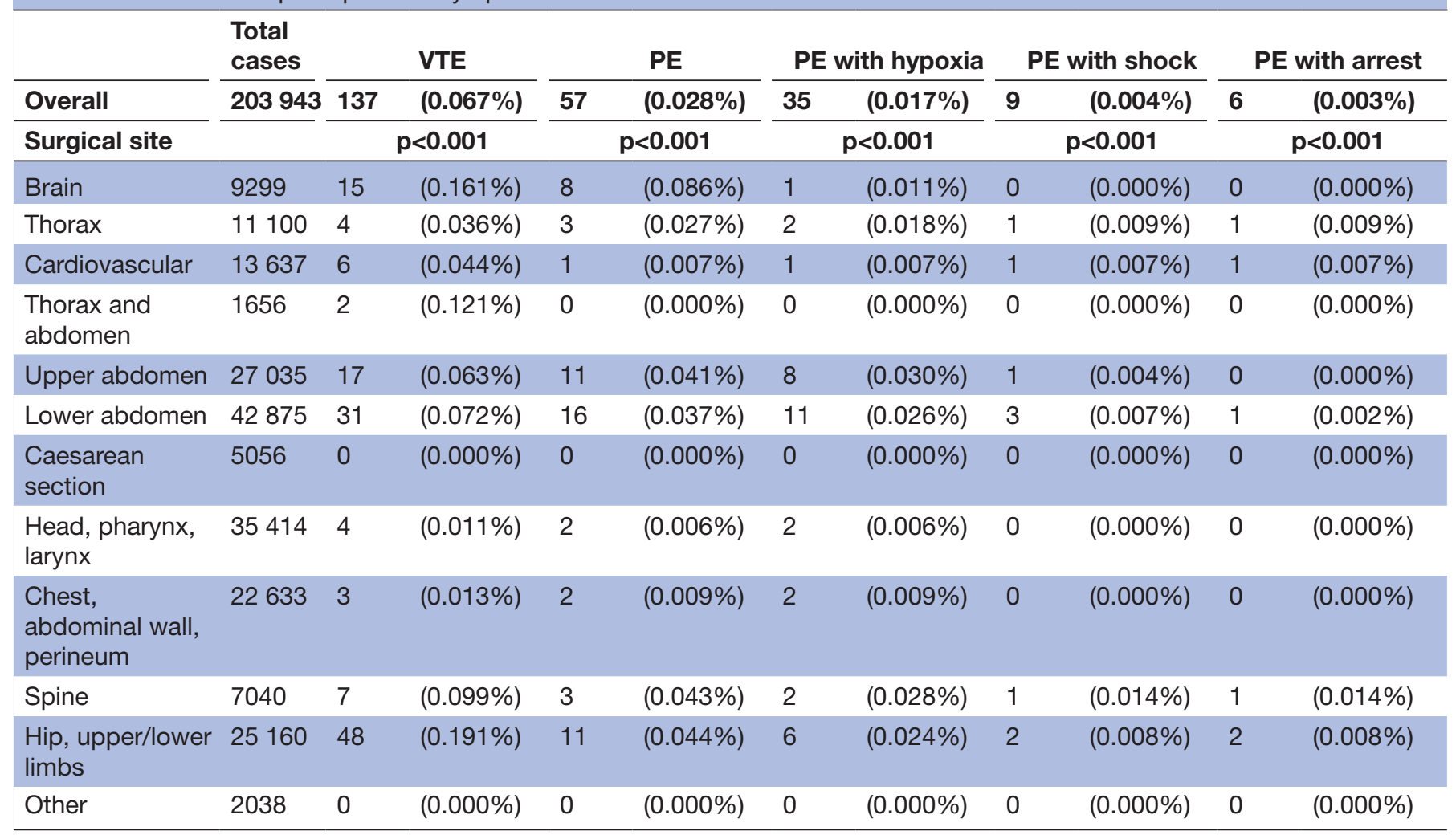

All data are described as numbers and percentages. The proportions of cases at each surgical site were compared using the $\chi^{2}$ test.

PE, pulmonary embolism; VTE, venous thromboembolism.

patients without a history of surgery within 2 months before VTE diagnosis, 293 patients were identified with postoperative symptomatic VTE during hospitalisation among all 29 centres of the COMMAND VTE Registry. Furthermore, 135 patients outside the eligible period and 21 patients who underwent surgery without the management by anaesthesiologists were excluded. Finally, the study population consisted of 137 patients diagnosed with VTE within 2 months after surgery, from 18 centres, between January 2010 and December 2013. The total number of surgical cases managed by anaesthesiologists during the study period in 18 centres was 203943.

\section{Incidence of postoperative symptomatic VTE}

The estimated incidence of postoperative symptomatic VTE was $0.067 \%(137 / 203943)$ and VTE with PE was $0.028 \%(57 / 203943)$ (table 1$)$. Of the 57 PE cases, 35 patients $(61.4 \%)$ had hypoxic symptoms, 9 patients $(15.8 \%)$ presented with shock and 6 patients $(10.5 \%)$ had cardiac arrest. As for the surgical site, the incidence of postoperative symptomatic VTE was relatively high in surgeries involving the brain, hip and upper/lower limbs. In terms of the types of anaesthesia, regional anaesthesia with or without general anaesthesia $(0.100 \%)$ was associated with a higher incidence of VTE than general anaesthesia alone $(0.045 \%)$ (table 1 and online supplemental table S1).

\section{Baseline characteristics and timing of VTE diagnosis}

Table 2 shows the demographic and clinical characteristics of patients with postoperative symptomatic VTE. Figure 2 presents the duration from the surgery to the diagnosis of postoperative symptomatic VTE. The median inter-quartile duration was 8 days (4-15 days); and 58 patients (42\%) were diagnosed within 7 days of surgery, while 79 patients $(58 \%)$ were diagnosed 7 days after the surgery. The greatest number of patients were diagnosed with VTE on postoperative day 8.

\section{Clinical outcomes after postoperative symptomatic VTE}

The cumulative incidence of recurrent VTE was $3.0 \%$ at the 30-day follow-up, $5.3 \%$ at the 90-day follow-up and $5.3 \%$ at the 5-year follow-up after postoperative symptomatic VTE (figure 3A). The cumulative incidence of major bleeding was $5.2 \%$ at 30 -day follow-up, $6.7 \%$ at the 90-day follow-up and $12.6 \%$ at the 5-year follow-up (figure 3B). The cumulative incidence of all-cause death was $3.7 \%$ at the 30 -day follow-up, $5.1 \%$ at the 90-day follow-up and $27.4 \%$ at the 5-year follow-up (figure 3C). The details of clinical events within 90 days are given in online supplemental table S2. VTE recurrence occurred in seven patients (4 patients were treated with anticoagulant therapy), all of which were early recurrences within 60 days of diagnosis. The difference in the cumulative incidence of recurrent VTE, major bleeding and all-cause 
Table 2 Baseline patients' characteristics

\begin{tabular}{|c|c|c|c|c|c|c|}
\hline & \multicolumn{2}{|c|}{ Total VTE } & \multicolumn{2}{|c|}{ PE with or without DVT } & \multicolumn{2}{|r|}{ DVT only } \\
\hline & \multicolumn{2}{|r|}{$\mathrm{N}=137$} & \multicolumn{2}{|c|}{$(\mathrm{N}=57)$} & \multicolumn{2}{|c|}{$(\mathrm{N}=80)$} \\
\hline \multicolumn{7}{|l|}{ Baseline characteristics } \\
\hline Age (years) & 66.2 & \pm 15.5 & 67.7 & \pm 12.6 & 65.1 & \pm 17.2 \\
\hline Men & 55 & $(40.1 \%)$ & 22 & $(38.6 \%)$ & 33 & $(41.3 \%)$ \\
\hline Body weight (kg) & 56.3 & \pm 11.8 & 57.8 & \pm 11.1 & 55.3 & \pm 12.3 \\
\hline Body mass index $\left(\mathrm{kg} / \mathrm{m}^{2}\right)$ & 23.2 & \pm 4.3 & 23.6 & \pm 3.7 & 22.9 & \pm 4.7 \\
\hline
\end{tabular}

\section{Baseline characteristics}

Surgical and anaesthesia characteristics

ASA PS

\begin{tabular}{|c|c|c|c|c|c|c|}
\hline ASA PS 1 & 19 & $(13.9 \%)$ & 10 & $(17.5 \%)$ & 9 & $(11.3 \%)$ \\
\hline ASA PS 2 & 91 & $(66.4 \%)$ & 42 & $(73.7 \%)$ & 49 & $(61.3 \%)$ \\
\hline ASA PS 3 & 22 & $(16.1 \%)$ & 4 & $(7.0 \%)$ & 18 & $(22.5 \%)$ \\
\hline ASA PS 4 & 5 & $(3.6 \%)$ & 1 & $(1.8 \%)$ & 4 & $(5.0 \%)$ \\
\hline Emergent surgery & 18 & $(13.1 \%)$ & 11 & $(19.3 \%)$ & 7 & $(8.8 \%)$ \\
\hline \multicolumn{7}{|l|}{ Surgical site } \\
\hline Hip and limb & 48 & (35.0\%) & 11 & $(19.3 \%)$ & 37 & $(46.3 \%)$ \\
\hline Brain & 15 & $(10.9 \%)$ & 8 & $(14.0 \%)$ & 7 & $(8.8 \%)$ \\
\hline Thorax and mediastinum & 4 & $(2.9 \%)$ & 3 & $(5.3 \%)$ & 1 & $(1.3 \%)$ \\
\hline Cardiovascular & 6 & $(4.4 \%)$ & 1 & $(1.8 \%)$ & 5 & $(6.3 \%)$ \\
\hline Thorax and abdomen & 2 & $(1.5 \%)$ & 0 & $(0.0 \%)$ & 2 & $(2.5 \%)$ \\
\hline Upper abdomen & 17 & $(12.4 \%)$ & 11 & $(19.3 \%)$ & 6 & $(7.5 \%)$ \\
\hline Lower abdomen & 31 & $(22.6 \%)$ & 16 & $(28.1 \%)$ & 15 & $(18.8 \%)$ \\
\hline Head and neck & 4 & $(2.9 \%)$ & 2 & $(3.5 \%)$ & 2 & $(2.5 \%)$ \\
\hline Chest abdominal wall and perineum & 3 & $(2.2 \%)$ & 2 & $(3.5 \%)$ & 1 & $(1.3 \%)$ \\
\hline Spine & 7 & $(5.1 \%)$ & 3 & $(5.3 \%)$ & 4 & $(5.0 \%)$ \\
\hline \multicolumn{7}{|l|}{ Type of anaesthesia } \\
\hline General anaesthesia & 61 & $(44.5 \%)$ & 26 & $(45.6 \%)$ & 35 & $(43.8 \%)$ \\
\hline General anaesthesia with regional anaesthesia & 56 & $(40.9 \%)$ & 24 & $(42.1 \%)$ & 32 & $(40.0 \%)$ \\
\hline Local anaesthesia & 20 & $(14.6 \%)$ & 7 & $(12.3 \%)$ & 13 & $(16.3 \%)$ \\
\hline \multicolumn{7}{|l|}{ Surgical position } \\
\hline Supine position & 100 & $(73.0 \%)$ & 39 & $(68.4 \%)$ & 61 & $(76.3 \%)$ \\
\hline Prone position & 6 & $(4.4 \%)$ & 2 & $(3.5 \%)$ & 4 & $(5.0 \%)$ \\
\hline Lateral position & 18 & $(13.1 \%)$ & 7 & $(12.3 \%)$ & 11 & $(13.8 \%)$ \\
\hline Lithotomy position & 11 & $(8.0 \%)$ & 8 & $(14.0 \%)$ & 3 & $(3.8 \%)$ \\
\hline Other position & 2 & $(1.5 \%)$ & 1 & $(1.8 \%)$ & 1 & $(1.3 \%)$ \\
\hline \multicolumn{7}{|l|}{ Comorbidities } \\
\hline Hypertension & 43 & $(31.4 \%)$ & 22 & $(38.6 \%)$ & 21 & $(26.3 \%)$ \\
\hline Diabetes mellitus & 15 & $(10.9 \%)$ & 7 & $(12.3 \%)$ & 8 & $(10.0 \%)$ \\
\hline Chronic kidney disease & 24 & $(17.5 \%)$ & 8 & $(14.0 \%)$ & 16 & $(20.0 \%)$ \\
\hline Dialysis & 2 & $(1.5 \%)$ & 0 & $(0.0 \%)$ & 2 & $(2.5 \%)$ \\
\hline History of chronic lung disease & 13 & $(9.5 \%)$ & 3 & $(5.3 \%)$ & 10 & $(12.5 \%)$ \\
\hline History of heart failure & 6 & $(4.4 \%)$ & 3 & $(5.3 \%)$ & 3 & $(3.8 \%)$ \\
\hline History of myocardial infarction & 4 & $(2.9 \%)$ & 0 & $(0.0 \%)$ & 4 & $(5.0 \%)$ \\
\hline History of stroke & 9 & $(6.6 \%)$ & 5 & $(8.8 \%)$ & 4 & $(5.0 \%)$ \\
\hline Atrial fibrillation & 8 & $(5.8 \%)$ & 7 & $(12.3 \%)$ & 1 & $(1.3 \%)$ \\
\hline Liver cirrhosis & 3 & $(2.2 \%)$ & 2 & $(3.5 \%)$ & 1 & $(1.3 \%)$ \\
\hline
\end{tabular}

Continued 
Table 2 Continued

\begin{tabular}{|c|c|c|c|c|c|c|}
\hline \multirow{3}{*}{ Connective tissue disease } & \multirow{2}{*}{\multicolumn{2}{|c|}{$\begin{array}{c}\text { Total VTE } \\
\mathrm{N}=137\end{array}$}} & \multirow{2}{*}{\multicolumn{2}{|c|}{$\begin{array}{l}\text { PE with or without DVT } \\
(\mathrm{N}=57)\end{array}$}} & \multirow{2}{*}{\multicolumn{2}{|c|}{$\begin{array}{c}\text { DVT only } \\
(\mathrm{N}=80)\end{array}$}} \\
\hline & & & & & & \\
\hline & 5 & (3.6\%) & 1 & (1.8\%) & 4 & (5.0\%) \\
\hline History of VTE & 1 & $(0.7 \%)$ & 1 & $(1.8 \%)$ & 0 & $(0.0 \%)$ \\
\hline History of major bleeding & 17 & $(12.4 \%)$ & 7 & $(12.3 \%)$ & 10 & $(12.5 \%)$ \\
\hline Active cancer & 41 & $(29.9 \%)$ & 24 & $(42.1 \%)$ & 17 & $(21.3 \%)$ \\
\hline Varicose vein & 8 & $(5.8 \%)$ & 3 & $(5.3 \%)$ & 5 & $(6.3 \%)$ \\
\hline Anticoagulants at VTE diagnosis & 14 & $(10.2 \%)$ & 6 & $(10.5 \%)$ & 8 & $(10.0 \%)$ \\
\hline Heparin & 6 & $(4.4 \%)$ & 3 & $(5.3 \%)$ & 3 & $(3.8 \%)$ \\
\hline Warfarin & 3 & $(2.2 \%)$ & 2 & $(3.5 \%)$ & 1 & $(1.3 \%)$ \\
\hline Direct oral anticoagulant & 5 & $(3.6 \%)$ & 1 & $(1.8 \%)$ & 4 & $(5.0 \%)$ \\
\hline \multicolumn{7}{|l|}{ Presentation } \\
\hline PE with hypoxemia & - & - & 35 & $(61.4 \%)$ & - & - \\
\hline PE with shock & - & - & 9 & $(15.8 \%)$ & - & - \\
\hline $\mathrm{PE}$ with cardiac arrest/collapse & - & - & 6 & $(10.5 \%)$ & - & - \\
\hline Proximal DVT & 64 & $(46.7 \%)$ & 21 & $(36.8 \%)$ & 43 & $(53.8 \%)$ \\
\hline \multicolumn{7}{|l|}{ Laboratory tests at diagnosis } \\
\hline Anaemia & 109 & $(82.6 \%)$ & 45 & (83.3\%) & 64 & (82.1\%) \\
\hline Thrombocytopenia & 6 & $(4.4 \%)$ & 5 & $(8.8 \%)$ & 1 & $(1.3 \%)$ \\
\hline eGFR $\left(\mathrm{mL} / \mathrm{min} / \mathrm{m}^{2}\right)$ & 78.3 & $(59.0-91.8)$ & 72.7 & $(51.2-87.7)$ & 80.4 & $(62.4-93.6)$ \\
\hline eGFR $<60 \mathrm{~mL} / \mathrm{min} / \mathrm{m}^{2}$ & 36 & $(26.3 \%)$ & 17 & $(29.8 \%)$ & 19 & $(23.8 \%)$ \\
\hline D-dimer ( $\mu \mathrm{g} / \mathrm{mL}, \mathrm{n}=122)$ & 16.5 & $(8.6-31.5)$ & 16.8 & $(8.6-39.3)$ & 16.4 & (7.9-27.3) \\
\hline Thrombophilia & 4 & $(2.9 \%)$ & 3 & $(5.3 \%)$ & 1 & $(1.3 \%)$ \\
\hline Initial anticoagulation therapy & 115 & (83.9\%) & 53 & $(93.0 \%)$ & 62 & (77.5\%) \\
\hline Heparin & 107 & $(78.1 \%)$ & 52 & $(91.2 \%)$ & 55 & $(68.8 \%)$ \\
\hline Fondaparinux & 11 & $(8.0 \%)$ & 2 & $(3.5 \%)$ & 9 & $(11.3 \%)$ \\
\hline Thrombolysis & 8 & $(5.8 \%)$ & 5 & $(8.8 \%)$ & 3 & $(3.8 \%)$ \\
\hline Inferior vena cava filter use & 26 & $(19.0 \%)$ & 13 & $(22.8 \%)$ & 13 & $(16.3 \%)$ \\
\hline Ventilator support & 6 & $(4.4 \%)$ & 6 & $(10.5 \%)$ & 0 & $(0.0 \%)$ \\
\hline Percutaneous cardiopulmonary support & 2 & $(1.5 \%)$ & 2 & $(3.5 \%)$ & 0 & $(0.0 \%)$ \\
\hline
\end{tabular}

Categorical variables are presented as numbers and percentages, and continuous variables are presented as the means and standard deviations or the medians and interquartile ranges based on their distributions. Chronic kidney disease was diagnosed if there was persistent proteinuria or if eGFR was $<60 \mathrm{~mL} / \mathrm{min} / 1.73 \mathrm{~m}^{2}$ for more than 3 months. The values of eGFR were calculated based on the equation reported by the Japan Association of Chronic Kidney Disease Initiative [man: $194^{\star} \mathrm{Scr}-1.094^{\star}$ age-0.287, woman: 194*Scr-1.094*age-0.287*0.739]. Anaemia was diagnosed if the value of haemoglobin was $<13 \mathrm{~g} / \mathrm{dL}$ for men and $<12 \mathrm{~g} / \mathrm{dL}$ for women. Thrombophilia includes protein $\mathrm{C}$ deficiency, protein S deficiency, antithrombin deficiency, and antiphospholipid syndrome.

ASA PS, American Society Anesthesiologists Performance Status; DVT, deep venous thrombosis; eGFR, estimated glomerular filtration rate; PE, pulmonary embolism; VTE, venous thromboembolism.

death was not significant between the PE-only and DVTonly groups, although the 30-day incidence of major bleeding and all-cause death was higher in the PE group than in the DVT-only group ( $11.1 \%$ vs $1.3 \%$ and $8.8 \%$ vs $0.0 \%$, respectively) (figure 4 ).

The cumulative 5-year incidence of recurrent VTE was not significantly different between patients with and without active cancer $(9.9 \%$ vs $3.3 \%$, log-rank $\mathrm{p}=0.13)$ (figure 5). In contrast, the cumulative 5-year incidences of major bleeding and all-cause death were significantly higher in patients with active cancer than in those without active cancer (major bleeding: $21.3 \%$ vs $8.8 \%$, log-rank $\mathrm{p}=0.046$, all-cause death: $45.5 \%$ vs $19.8 \%$, logrank $\mathrm{p}=0.001$ ) (figure 5).

\section{DISCUSSION}

The main findings of this study are as follows: (1) the incidence of postoperative symptomatic VTE within 2 months after surgery was $0.067 \%$ and VTE with PE was $0.028 \%$, representing 203943 patients from 18 centres in Japan; (2) the incidence of postoperative symptomatic 


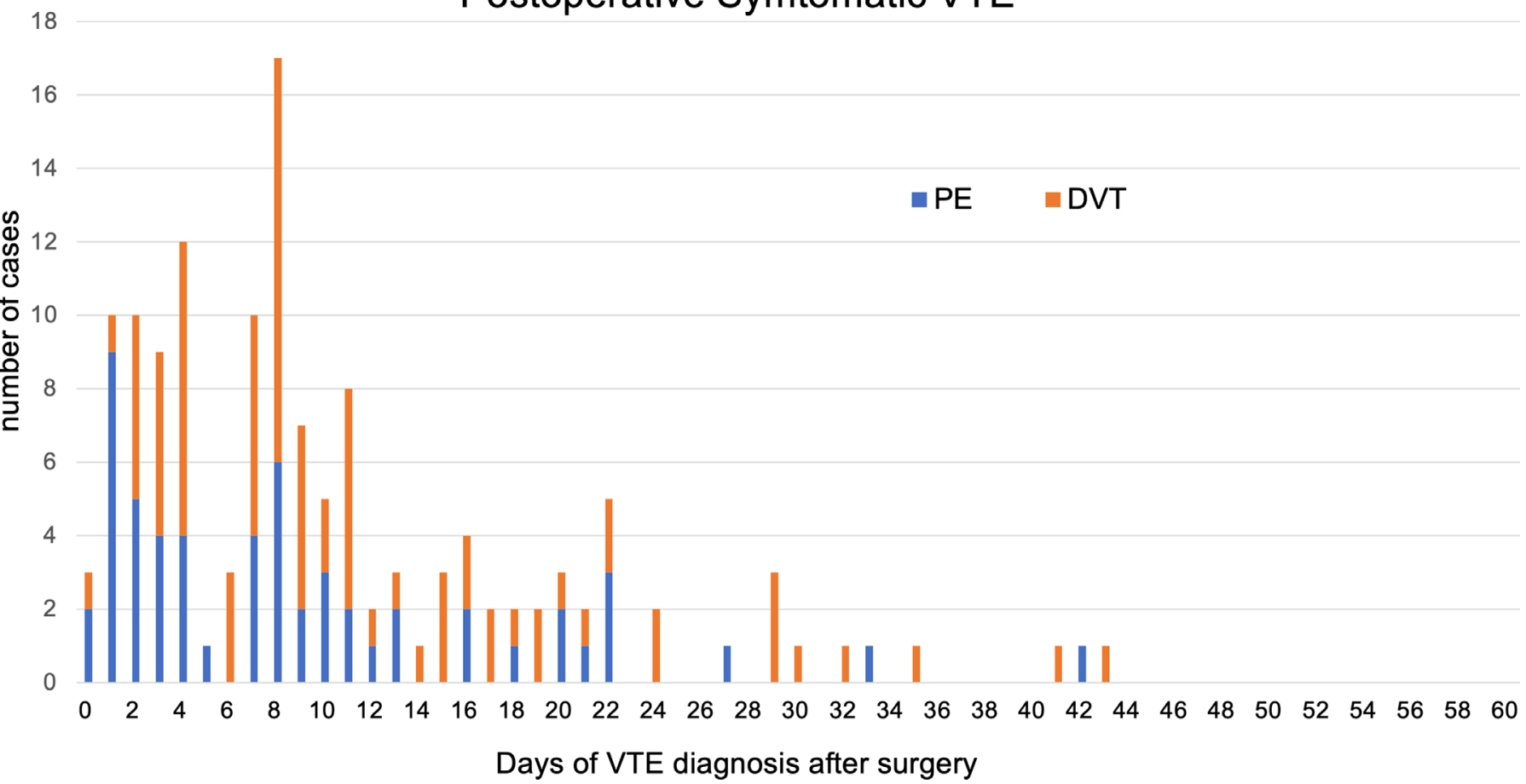

Figure 2 The distribution of days of VTE diagnosis after surgery. DVT, deep vein thrombosis; PE, pulmonary embolism; VTE, venous thromboembolism.

VTE varied widely, according to surgical and anaesthetic characteristics and (3) nearly half of the patients were diagnosed within 7 days of the surgery, while the rest were diagnosed 7 days after surgery, with the highest number of patients diagnosed on postoperative day 8 .

The strength of the present study is that the diagnosis of symptomatic VTE was accurately diagnosed by cardiologists (specialists for VTE in Japan), and the detailed information about this postoperative complication and its long-term prognosis could be evaluated, in contrast to previous studies on the subject.

VTE is considered relatively rare in Asian people and the small number of cases makes epidemiological studies difficult to perform. ${ }^{11}$ Previously, three major studies from Japan had evaluated the incidence of the postoperative complication of VTE. ${ }^{1018}$ The first study was based on the JSA-initiated questionnaire annual survey, where the incidence of PE was $0.031 \%(3667 / 11786489) .{ }^{10}$ The second study used the diagnosis-procedure combination (DPC) database, and the incidence of VTE and PE was $0.24 \%$ (2485/1 016496$)$ and $0.05 \%$ (538/1 016 496), respectively. ${ }^{18}$ The third study used the National Clinical Database (NCD), a nationwide project linked to the surgical board certification system. The incidence of DVT and PE was $0.26 \%(984 / 382124)$ and $0.14 \%$ (553/382 124), respectively. ${ }^{19}$ The incidence of postoperative
(A) Recurrent VTE

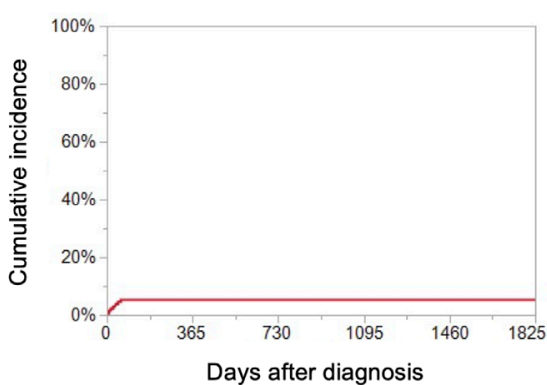

\begin{tabular}{lcccccc}
\hline & 0-day & 7 -day & 30-day & 90 -day & 1-year & 5-year \\
\hline Nof patients with event & & 1 & 4 & 7 & 7 & 7 \\
Nof patients at risk & 137 & 134 & 129 & 124 & 116 & 32 \\
Cumulative incidence & & $0.8 \%$ & $3.0 \%$ & $5.3 \%$ & $5.3 \%$ & $5.3 \%$ \\
\hline
\end{tabular}

(B) Major bleeding
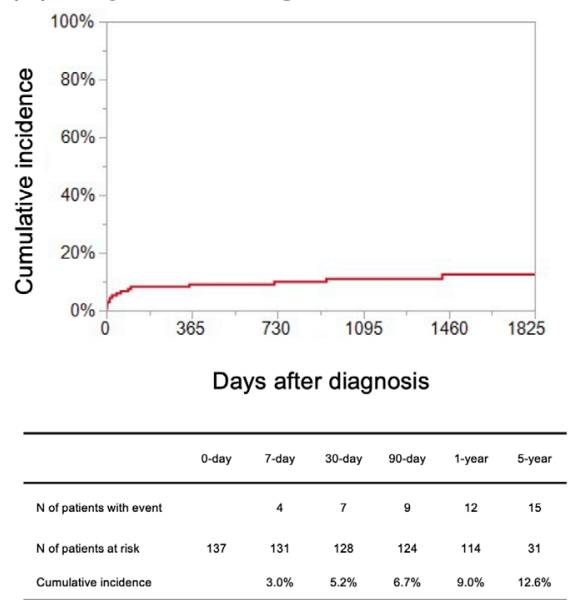

(c) All-cause death

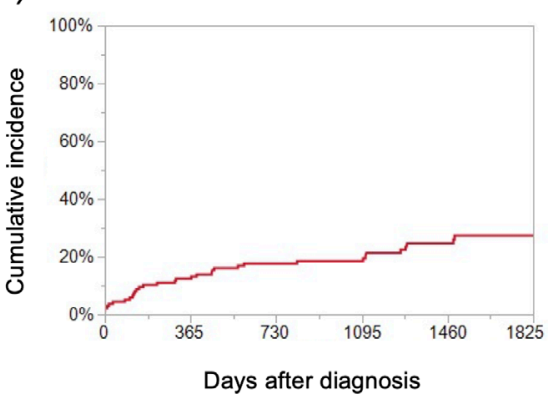

\begin{tabular}{lcccccc}
\hline & 0-day & 7-day & 30-day & 90 -day & 1-year & 5-year \\
\hline N of patients with event & & 3 & 5 & 7 & 17 & 33 \\
N of patents at risk & 137 & 136 & 133 & 131 & 121 & 34 \\
Cumulative incidence & & $2.2 \%$ & $3.7 \%$ & $5.1 \%$ & $12.4 \%$ & $27.4 \%$ \\
\hline
\end{tabular}

Figure 3 The Kaplan-Meier curves for the clinical events after VTE diagnosis. (A) Recurrent VTE, (B) major bleeding and (C) allcause death. VTE, venous thromboembolism. 


\section{(A) Recurrent VTE}

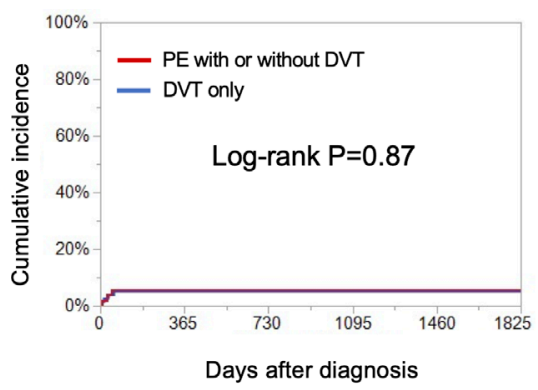

\begin{tabular}{lcccccc}
\hline & 0-day & 7-day & 30-day & 90-day & 1-year & 5-year \\
\hline PE with or without DVT & & & & & & \\
\hline N Of patients with event & & 0 & 2 & 3 & 3 & 3 \\
N of patients at risk & 57 & 56 & 51 & 50 & 47 & 16 \\
Cumulative incidence & & $0.0 \%$ & $3.8 \%$ & $5.7 \%$ & $5.7 \%$ & $5.7 \%$ \\
\hline DVT only & & & & & & \\
\hline N of patients with event & & 1 & 2 & 4 & 4 & 4 \\
N of pationts at risk & 80 & 80 & 79 & 75 & 70 & 17 \\
Cumulative incidence & & $1.3 \%$ & $2.5 \%$ & $5.0 \%$ & $5.0 \%$ & $5.0 \%$ \\
\hline
\end{tabular}

(B) Major bleeding
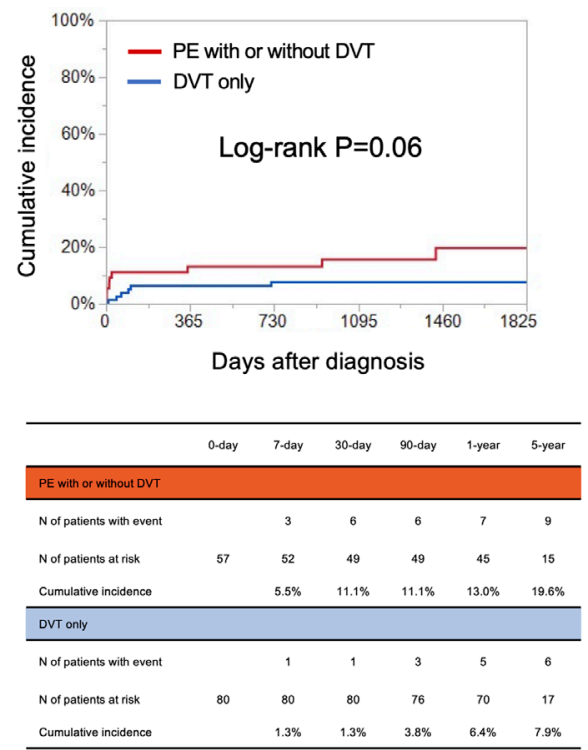

(c) All-cause death
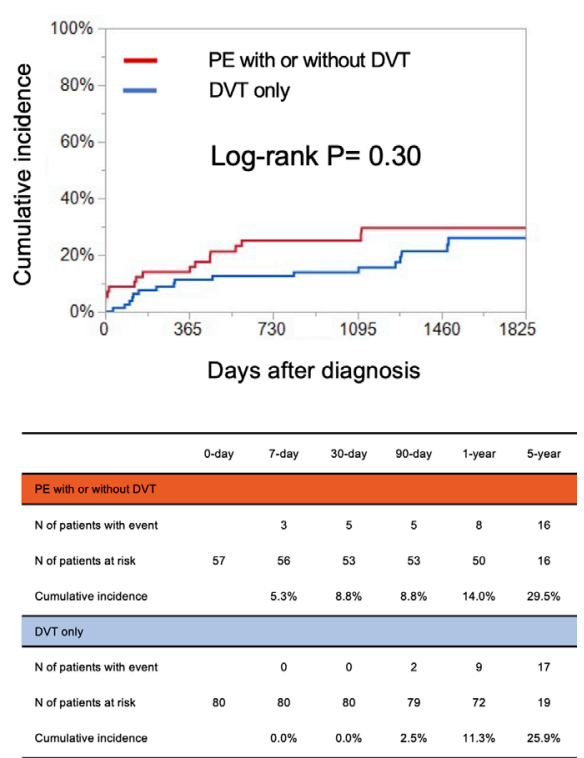

Figure 4 The Kaplan-Meier curves for the clinical events after VTE diagnosis comparing PE and DVT. (A) Recurrent VTE, (B) major bleeding and (C) all-cause death. DVT, deep vein thrombosis; PE, pulmonary embolism; VTE, venous thromboembolism.

symptomatic VTE in the current study was lower than that in the DPC study. In the DPC study, VTE was identified based on the International Classification of Diseases, 10th version codes; and; therefore, it may have been misclassified and over-rated. In this study, we used data on symptomatic VTE confirmed by cardiologists. This may explain the lower incidence compared with the NCD study, which included asymptomatic VTE. The incidence of postoperative VTE was reported to be $0.58 \%-2.2 \%$, based on the clinical databases in the USA. Therefore, postoperative VTE incidence was suggested to be lower in Japan than in the USA and Europe. These differences could be explained by ethnic variations. ${ }^{11}$ Western guidelines, ${ }^{45}$ due to racial disparities, are more likely to lead to overtriage in the Japanese population.

Another important feature of the current study was the comparison of the incidence of postoperative symptomatic VTE across surgical sites. Similar to the JSA initiated questionnaire study, ${ }^{10}$ neurosurgeries and orthopaedic surgeries (hip, upper and lower extremity) were associated with a higher incidence of postoperative symptomatic VTE. According to the Japanese guidelines, there is

\section{(A) Recurrent VTE}
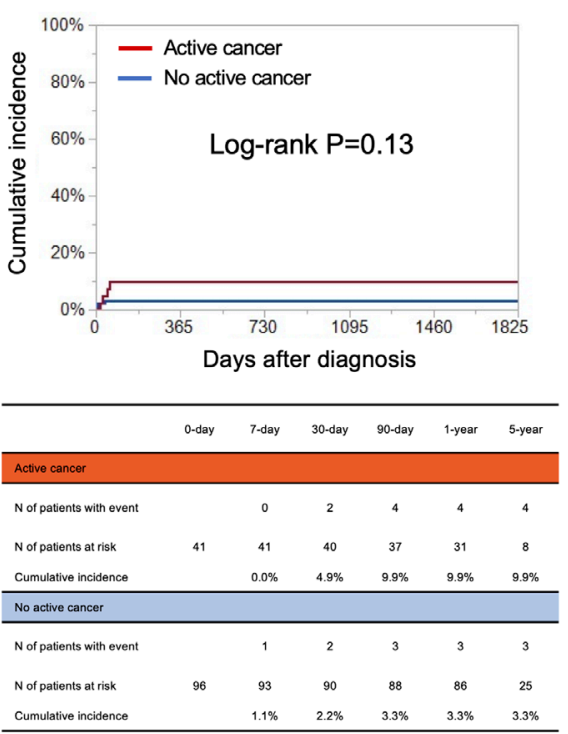

(B) Major bleeding
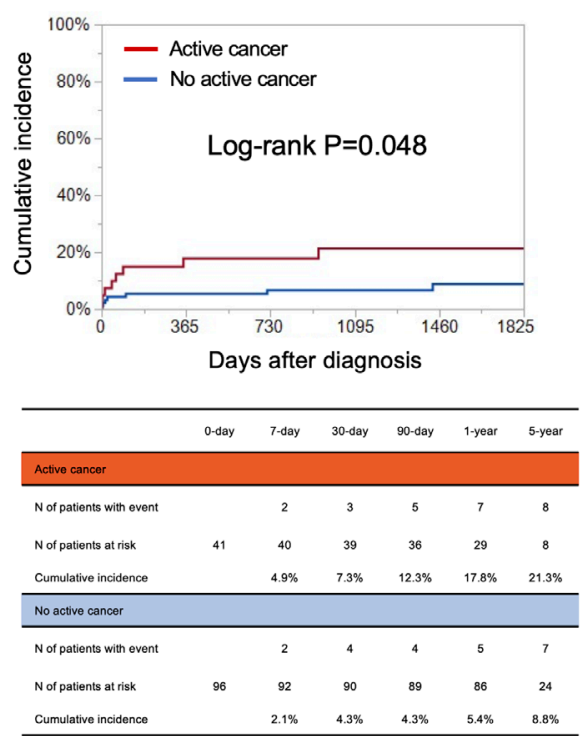

\section{(c) All-cause death}
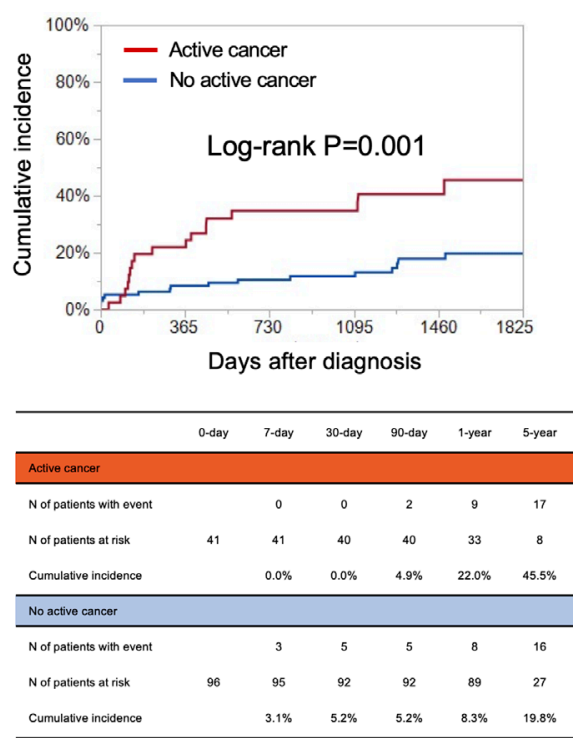

Figure 5 The Kaplan-Meier curves for clinical events after VTE diagnosis with and without active cancer. (A) Recurrent VTE, (B) major bleedingand (C) all-cause death. DVT, deep vein thrombosis; PE, pulmonary embolism; VTE, venous thromboembolism. 
a high risk of postoperative symptomatic VTE in patients over 40 years of age undergoing major cancer surgery; however, in the present study, abdominal surgery was not identified with high risk. Therefore, risks should be stratified according to the surgical sites and procedures, and the additional risks in each patient should be considered in the preventive strategies.

Additionally, in this study, the timing of the onset of postoperative symptomatic VTE was bimodal. These results may suggest that postoperative symptomatic VTE occurs, not only in the very acute postoperative period, which is directly affected by surgical immobilisation, but also approximately 10 days after surgery; the information can guide the healthcare providers involved in surgery, regarding the risk perception and diagnosis of postoperative symptomatic VTE.

The duration of anticoagulant therapy is generally divided into an initial treatment phase (up to 7 days), a maintenance treatment phase $(\sim 3$ months after the initial treatment) and prolonged treatment phase (beyond 3 months).$^{20}$ Surgery is a transient risk factor for VTE; prolonged treatment is usually not performed, as the possibility of recurrence is considered relatively low. In this study, VTE recurrence had occurred in all seven affected patients within 3 months of the onset, and no recurrence was observed after 3 months, suggesting the importance of relatively early recurrence.

PE was apparently associated with a higher mortality, especially in the early phase of postoperative symptomatic VTE, although the difference between the PE-only and DVT-only groups was not significant. This difference may be explained by the insufficient sample size. Notably, the initial mortality rate and recurrence rate were higher for acute PE than for DVT. ${ }^{21} 22$ Therefore, in comparison to DVT, postoperative PE should be more closely monitored and aggressively treated. ${ }^{3}$

\section{Study limitations}

First, two different databases were combined to estimate the incidence of postoperative symptomatic VTE. Although the COMMAND Registry included real consecutive patients with acute symptomatic VTE, ${ }^{10}$ for determining VTE incidence, we included only the cases in which intraoperative management was performed by an anaesthesiologist. Second, patients outside the eligible period in the COMMAND VTE Registry were also excluded, which may have influenced the results of this study. As a certain number of patients were excluded due to ineligible centres, the incidence of postoperative symptomatic VTE could have been greatly influenced, especially as the analysis targeted low event rates. Third, this was a retrospective cohort study with inherent limitations based on the observational study design. In particular, the prophylactic and therapeutic management for postoperative symptomatic VTE were based on the discretion of the attending physicians, which may have influenced the clinical outcomes. However, in the COMMAND Registry, the definitions of VTE were specified in advance, and the follow-up after VTE was nearly complete. Fourth, the incidence of postoperative symptomatic VTE may depend on the status of VTE prophylaxis. However, the JSA annual report does not include data on prophylaxis status, and we could not determine this status for the entire study population. Fifth, the JSA annual report does not include data on the status of malignancy either, and we could not determine it for the entire study population. Finally, we also considered the postoperative date of onset, but the disease may have developed before the surgery or the diagnosis. Nevertheless, we do not expect a significant gap between the onset and diagnosis, because we included only symptomatic patients with postoperative VTE.

\section{Conclusions}

This study, combining the large real-world VTE database and anaesthetic database in Japan, revealed the incidence, clinical features and prognosis of postoperative symptomatic VTE, providing useful information for all healthcare providers involved in various surgeries.

\section{Author affiliations}

${ }^{1}$ Department of Anesthesia, Kyoto University Hospital, Kyoto, Japan

${ }^{2}$ Department of Cardiovascular Medicine, Kyoto University Graduate School of

Medicine, Faculty of Medicine, Kyoto, Japan

${ }^{3}$ Department of Pharmacoepidemiology, Graduate School of Medicine and Public Health, Kyoto University, Kyoto, Japan

${ }^{4}$ Department of Clinical Anesthesiology, Mie University Graduate School of Medicine Faculty of Medicine, Tsu, Mie, Japan

Acknowledgements We appreciate the support and collaboration of the co-investigators participating in the COMMAND VTE Registry. We also thank the following doctors: Hiroshi Miyawaki, Takehiko Adachi, Tsutomu Shichino, Shinichi Hamasaki, Shinichi Nakao, Jun Utumi, Kouichi Kitou, Toshiaki Mochizuki, Makoto Okamura, Kazuo Shindo, Jun-ichirou Yokoyama, Yoshito Shiraishi, Hiroyuki Mima, Keiji Tanimoto, Takeshi Kato, Toyohiko Ohigashi, Satoshi Takabuchi, Tetsutaro Shinomura for extracting the JSA annual report and the additional data from each centre.

Contributors СT: This author had full access to all the data in the study and take responsibility for the integrity of the data and the accuracy of the data analysis. This author helped design and conduct the study, analyse the data, and write and revise the manuscript. YY: This author also had full access to all the data in the study and take responsibility for the integrity of the data and the accuracy of the data analysis. This author helped design and conduct the study, analyse the data, and write and revise the manuscript. MT: This author helped analyse the data and write and revise the manuscript. HY: This author helped analyse the data and write and revise the manuscript. LD: This author helped analyse the data and write the manuscript. $\mathrm{MH}$ : This author helped analyse the data and write and revise the manuscript. AH: This author helped analyse the data and write and revise the manuscript. KO: This author helped analyse the data and write and revise the manuscript. KK: This author helped analyse the data and write and revise the manuscript. KF: This author helped design and conduct the study and write the manuscript. TaM: This author helped design and conduct the study, analyse the data, and write and revise the manuscript. TK: This author helped conduct the study, analyse the data and write and revise the manuscript. ToM: This author helped design and conduct the study, analyse the data, write and revise the manuscript, and guarantor.

Funding This work was supported in part by the JSPS KAKENHI (grant number 20K09242; TM, principal investigator). The COMMAND VTE Registry is supported by an independent clinical research organisation (Research Institute for Production Development, Kyoto, Japan) and research funding from Mitsubishi Tanabe Pharma Corporation. The research funding had no role in the design and conduct of the study; collection, management, analysis, and interpretation of the data; and preparation, review, or approval of the manuscript.

Competing interests Dr. Yamashita received lecture fees from Daiichi-Sankyo, Bristol-Myers Squibb, Pfizer and Bayer Healthcare. Dr. Morimoto received lecture 
fees from Mitsubishi Tanabe Pharma and Pfizer Japan and consultant fees from Asahi Kasei, Bristol-Myers Squibb and Boston Scientific. Dr. Kawakami received consulting fees from Kaken Pharmaceutical Co., Ltd.; research funds from Sumitomo Dainippon Pharma Co., Ltd., Bayer Yakuhin Ltd., Stella Pharma Corporation, CMIC Co., Ltd., and Pfizer Japan Inc.; honorarium from Daiichi-Sankyo Co., Ltd., Mitsubishi Tanabe Pharma Corporation, AbbVie GK, Takeda Pharmaceutical Co., Ltd., Mitsubishi Chemical Holdings Corporation, and Astra Zeneca; and holds stocks of Real-World Data Co., Ltd. All other authors have reported that they have no relationships relevant to the contents of this paper to disclose.

Patient consent for publication Not applicable.

Ethics approval This retrospective observational study was conducted according to the STrengthening the Reporting of OBservational studies in Epidemiology (STROBE) guidelines. This study was approved by the Ethics Committee of the Kyoto University Hospital, Kyoto, Japan (approval number: R1822, 18 December 2018; Chairperson Prof Shinji Kosugi). Following Ethics Committee approval, additional data, including the JSA annual reports, were collected from the centres listed in the Command VTE Registry, from March 2019 to September 2019. Written informed consent from each patient was waived, because we used clinical information obtained in routine clinical practice. This method is concordant with the guidelines for epidemiological studies issued by the Ministry of Health, Labor and Welfare in Japan.

Provenance and peer review Not commissioned; externally peer reviewed.

Data availability statement Data available on request from the authors. The data that support the findings of this study are available from Chikashi Takeda or Yugo Yamashita, upon reasonable request. The data that support the findings of this study are available from the corresponding author, upon reasonable request.

Supplemental material This content has been supplied by the author(s). It has not been vetted by BMJ Publishing Group Limited (BMJ) and may not have been peer-reviewed. Any opinions or recommendations discussed are solely those of the author(s) and are not endorsed by BMJ. BMJ disclaims all liability and responsibility arising from any reliance placed on the content. Where the content includes any translated material, BMJ does not warrant the accuracy and reliability of the translations (including but not limited to local regulations, clinical guidelines, terminology, drug names and drug dosages), and is not responsible for any error and/or omissions arising from translation and adaptation or otherwise.

Open access This is an open access article distributed in accordance with the Creative Commons Attribution Non Commercial (CC BY-NC 4.0) license, which permits others to distribute, remix, adapt, build upon this work non-commercially, and license their derivative works on different terms, provided the original work is properly cited, appropriate credit is given, any changes made indicated, and the use is non-commercial. See: http://creativecommons.org/licenses/by-nc/4.0/.

\section{ORCID iDs}

Chikashi Takeda http://orcid.org/0000-0002-0903-0201

Hiroshi Yonekura http://orcid.org/0000-0003-0523-2997

Koji Kawakami http://orcid.org/0000-0002-7477-4071

Takeshi Morimoto http://orcid.org/0000-0002-6844-739X

Takeshi Kimura http://orcid.org/0000-0002-5665-4076

Toshiyuki Mizota http://orcid.org/0000-0003-2770-4262

\section{REFERENCES}

1 Lindblad B, Eriksson A, Bergqvist D. Autopsy-verified pulmonary embolism in a surgical department: analysis of the period from 1951 to 1988. Br J Surg 1991;78:849-52.

2 Office of the Surgeon General. The surgeon general's call to action to prevent deep vein thrombosis and pulmonary embolism. Rockville, MD, USA: Office of the Surgeon General, 2008.

3 JCS Joint Working Group. Guidelines for the diagnosis, treatment and prevention of pulmonary thromboembolism and deep vein thrombosis (JCS 2009). Circ J 2011;75:1258-81.
4 Falck-Ytter Y, Francis CW, Johanson NA, et al. Prevention of VTe in orthopedic surgery patients: antithrombotic therapy and prevention of thrombosis, 9th ED: American College of chest physicians evidence-based clinical practice guidelines. Chest 2012;141:e27 $8 \mathrm{~S}-325$

5 Gould MK, Garcia DA, Wren SM, et al. Prevention of VTe in nonorthopedic surgical patients: antithrombotic therapy and prevention of thrombosis, 9th ED: American College of chest physicians evidence-based clinical practice guidelines. Chest 2012;141:e227S-77.

6 Bouras G, Burns EM, Howell A-M, et al. Risk of post-discharge venous thromboembolism and associated mortality in general surgery: a population-based cohort study using linked hospital and primary care data in England. PLoS One 2015;10:e0145759.

7 Gangireddy C, Rectenwald JR, Upchurch GR, et al. Risk factors and clinical impact of postoperative symptomatic venous thromboembolism. J Vasc Surg 2007;45:335-42.

8 Colorectal Writing Group for Surgical Care and Outcomes Assessment Program-Comparative Effectiveness Research Translation Network (SCOAP-CERTAIN) Collaborative, Nelson DW, Simianu VV, et al. Thromboembolic complications and prophylaxis patterns in colorectal surgery. JAMA Surg 2015;150:712-20.

9 Groot OQ, Ogink PT, Paulino Pereira NR, et al. High risk of symptomatic venous thromboembolism after surgery for spine metastatic bone lesions: a retrospective study. Clin Orthop Relat Res 2019;477:1674-86.

10 Kuroiwa M, Morimatsu H, Tsuzaki K, et al. Changes in the incidence, case fatality rate, and characteristics of symptomatic perioperative pulmonary thromboembolism in Japan: results of the 2002-2011 Japanese Society of Anesthesiologists perioperative pulmonary thromboembolism (JSA-PTE) study. J Anesth 2015;29:433-41.

11 Montagnana M, Favaloro EJ, Franchini M, et al. The role of ethnicity, age and gender in venous thromboembolism. J Thromb Thrombolysis 2010;29:489-96.

12 Liew NC, Moissinac K, Gul Y. Postoperative venous thromboembolism in Asia: a critical appraisal of its incidence. Asian J Surg 2003;26:154-8.

13 Wang K-L, Yap ES, Goto S, et al. The diagnosis and treatment of venous thromboembolism in Asian patients. Thromb J 2018:16:4

14 Wendelboe AM, McCumber M, Hylek EM, et al. Global public awareness of venous thromboembolism. J Thromb Haemost 2015;13:1365-71.

15 Yamashita $\mathrm{Y}$, Morimoto $\mathrm{T}$, Amano $\mathrm{H}$, et al. Anticoagulation therapy for venous thromboembolism in the real world - From the COMMAND VTE registry. Circ J 2018;82:1262-70.

16 Laporte S, Mismetti P, Décousus $\mathrm{H}$, et al. Clinical predictors for fatal pulmonary embolism in 15,520 patients with venous thromboembolism: findings from the Registro Informatizado de la Enfermedad TromboEmbolica venosa (RIETE) registry. Circulation 2008;117:1711-6.

17 Schulman S, Angerås U, Bergqvist D, et al. Definition of major bleeding in clinical investigations of antihemostatic medicinal products in surgical patients. J Thromb Haemost 2010;8:202-4.

18 Kunisawa S, Ikai H, Imanaka Y. Incidence and prevention of postoperative venous thromboembolism: are they meaningful quality indicators in Japanese health care settings? World J Surg 2012;36:280-6.

19 Hata T, lkeda M, Miyata $\mathrm{H}$, et al. Frequency and risk factors for venous thromboembolism after gastroenterological surgery based on the Japanese National Clinical Database (516 217 cases). Ann Gastroenterol Surg 2019;3:534-43.

20 Kearon C, Akl EA, Comerota AJ, et al. Antithrombotic therapy for VTe disease: antithrombotic therapy and prevention of thrombosis, 9th ED: American College of chest physicians evidence-based clinical practice guidelines. Chest 2012;141:e419S-96.

21 Murin S, Romano PS, White RH. Comparison of outcomes after hospitalization for deep venous thrombosis or pulmonary embolism. Thromb Haemost 2002;88:407-14.

22 Nakamura M, Miyata T, Ozeki Y, et al. Current venous thromboembolism management and outcomes in Japan. Circ $J$ 2014;78:708-17. 